\title{
Leg-drive Does Not Affect Upper Extremity Muscle Activation during a Bench Press Exercise
}

\author{
Jacob K. Gardner, ${ }^{1, *}$, Justin T. Chia ${ }^{2}$, Kelsey L. Miller ${ }^{1}$ \\ ${ }^{1}$ Department of Kinesiology and Health Science, Biola University, USA \\ ${ }^{2}$ Department of Health and Human Development, Western Washington University, USA
}

Copyright $\odot 2019$ by authors, all rights reserved. Authors agree that this article remains permanently open access under the terms of the Creative Commons Attribution License 4.0 International License

\begin{abstract}
The purpose of this study was to determine if muscle activity of upper extremity muscles differed depending on the involvement of the legs during a bench press. The study included 15 male and 12 female recreationally trained, college age participants. There were 2 testing sessions. Session 1: Participants performed a 1-repetition maximum in a standard bench press followed by a leg-drive familiarization. For the familiarization, participants were instructed in the leg-drive technique (buttocks remained on the bench) and given ample time to practice until comfortable and which satisfied the researchers. Leg-drive pressing force was verified by measuring anterior-posterior and vertical ground reaction forces measured by a force plate. Session 2: Outfitted with surface electromyography (EMG), participants performed 3 repetitions in the bench press with $75 \%$ of the standard bench press 1-repetition maximum (1RM) under three conditions: 1) standard 2) leg-drive, 3) legs-in-the-air. The normalized average of the peaks of the three reps for each muscle were analyzed using a 2 × 3 (gender $\mathrm{x}$ condition) ANOVA. The ANOVA revealed no significant differences across groups or conditions (pectoralis major $\mathrm{p}=0.405$, anterior deltoid $p=0.297$, triceps brachii $p=0.092$ ). When comparing a standard bench press to leg-drive, our results indicate that similar amounts of muscle activation are required for the task regardless of the leg involvement. This work indicates that using a leg-drive technique that does not allow the buttocks to rise from the bench, does not result in a change in upper extremity muscle activity compared to a standard bench press. Due to lack of differences across conditions, athletes and strength coaches should base their decision on the use of leg-drive on their personal preference.
\end{abstract}

Keywords Lower-extremity, Electromyography, Resistance Training, Muscle Contraction

\section{Introduction}

Resistance exercise is an essential component to the training regime of athletes and the general public alike. The bench press is commonly used as a measure of upper-body strength with the prime movers being the pectoralis major, the triceps brachii, and the anterior deltoids (1). While it is clear that the bench press is an upper-body exercise, it is less clear to what extent the lower extremities are, or should be, involved in the exercise. Professional organizations such as the National Strength and Conditioning Association instruct that the left and right feet of the lifter are to remain on the floor during the lift for stability as 2 (out of 5) points of contact (13). Anecdotal evidence in weight rooms suggest that it may be beneficial to use the lower extremities as a performance enhancing driving mechanism in addition to stability. To accomplish such a task, lifters flex at the knee to move the foot behind a vertical plane at the knee joint in order to press with their legs (leg-drive) while performing the lift.

Observationally, many weight-training participants indicate that leg-drive while performing the bench press gives them a sense of improved performance. In an article about inter-subject variability of muscle synergies during a bench press, authors Kristiansen et al. discovered that experienced power lifters utilized the vastus lateralis muscle while lifting $75 \%$ of a 3 repetition maximum (RM) load (8). Interestingly, untrained individuals in the same study did not exhibit this same muscle activation pattern in the lower extremities. The authors suggested that the use of the vastus lateralis in experienced lifters acts to isometrically extend the knee, resulting in increased stability and stiffness of the torso, allowing greater strength expression (8). However, these authors did not control for participant posture and it was noted that the experienced lifters tended to arch their backs during the lift. Thus, the increased vastus lateralis activation could partly be due to driving the legs but also could be due to the effort required to maintain an arched position. 
Little other scientific evidence exists on the effects of leg-drive on bench press performance. Therefore, the purpose of this study was to compare muscle activation of the pectoralis major, anterior deltoid, and triceps brachii muscles during three bench press conditions. The three conditions included: 1 . Feet on ground but not driving (standard (13), figure 1a), 2. Feet on ground and driving (leg-drive, figure 1b), and 3. Feet off the ground with hips and knees flexed to 90 degrees (feet-in-the-air, figure 1c). Due to limited literature on leg-involvement during a bench press, it was hypothesized that no differences in muscle activation of the three upper extremity muscles would exist regardless of lower extremity involvement.

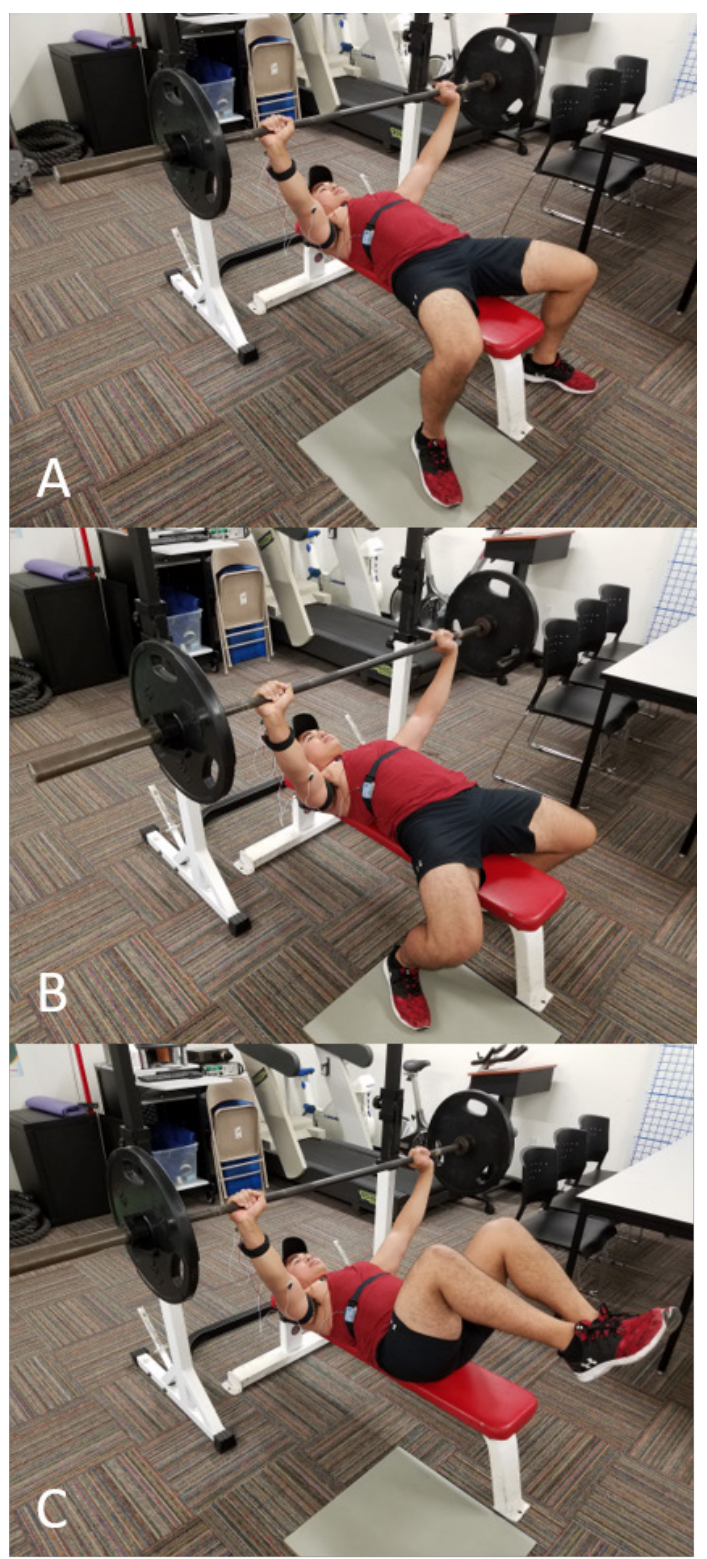

Figure 1. (A) Standard bench press condition. (B) Leg-drive condition. Note that during the leg-drive, participants aligned their feet behind the vertical line of the knees while still maintaining a flat foot on the floor. (C) Feet-in-the-air condition. Note that the hips and knees are flexed to approximately 90 degrees.

\section{Methods}

\subsection{Approach to the Problem}

The protocol was conducted on two separate days, 1 week apart. Participants were asked to refrain from physical activity 2 days prior to and during the experiment to avoid cumulative fatigue. On the first day, a bench press 1RM protocol (4) was conducted to determine the maximum weight the participant could lift in the bench press exercise. The 1RM was only performed in the standard bench press condition. A standardized five-point body contact position was utilized for the 1RM protocol (13). On day 1, participants were familiarized with the leg-drive technique and metronome pacing.

On day two, participants performed a maximal voluntary isometric contraction (MVIC) for the purpose of EMG normalization followed by 3 bench press reps in the 3 testing conditions. It should be noted that all three lifting styles are common and are safe for the lifter to perform. Before MVIC testing, surface EMG electrodes (Noraxon Dual $\mathrm{Ag} / \mathrm{AgCl}$ electrodes with inter-electrode distance of $1.75 \mathrm{~cm}$, Noraxon USA Inc., Scottsdale, AZ) were placed over the muscle bellies of the pectoralis major (sternal fibers), anterior deltoid, and lateral head of the triceps brachii on the right side of the body according to the SENIAM project guidelines (5).

\subsection{Subjects}

Fifteen males (age $22.1 \pm 2.1 \mathrm{yrs}$, height $1.71 \pm 0.04 \mathrm{~m}$, mass $74.7 \pm 6.4 \mathrm{~kg}, 1 \mathrm{RM} 103.56 \pm 24.11 \mathrm{~kg}$ ) and twelve females (age $20.5 \pm 1.3 \mathrm{yrs}$, height $1.63 \pm 0.07 \mathrm{~m}$, mass $60.1 \pm 11.3 \mathrm{~kg}, 1 \mathrm{RM} 48.10 \pm 5.65 \mathrm{~kg}$ ) over the age of 18 participated in this study. Individuals were considered for inclusion had they been regularly resistance training with the bench press exercise for at least 6 months prior to the start of the study while also sustaining no serious lower or upper body injuries during this time. Participants were informed of the benefits and risks of the investigation prior to signing an informed consent approved by the University's Protection of Human Rights in Research Committee (approval number F16-021_SE). This research was conducted in accordance with the 1964 Declaration of Helsinki.

\subsection{Procedures}

In the setup for the leg-drive, participants maintained five points of contact during the press but flexed their knees, causing the feet to align behind the knees while remaining flat on the floor (Figure 1a). The participants were instructed to drive their feet down and forward as if performing an isometric knee extension into the floor. It was emphasized not to allow the buttocks to rise off the bench during the leg-drive. Participants practiced until 
comfortable and the investigators were satisfied.

To verify that the participants were driving the legs as desired, the right foot was placed on a force platform (1200 Hz, Advanced Mechanical Technology Inc. (AMTI), Watertown, MA) and the vertical and anterior-posterior ground reaction forces (GRF) were recorded (AMTI Netforce, Watertown, MA). During the zeroing process, the participant maintained contact with the force plate without pressing. The force plate was zeroed with the foot in place to remove the weight of the leg from the GRF data. A positive vertical and positive antero-posterior GRF indicated a successful leg-drive.

To minimize compounding effects of delayed onset muscle soreness (DOMS), testing days were separated by 1 week. Each EMG site was shaved (if applicable), abraded with sandpaper, cleansed with alcohol, and treated with an electrode skin prep gel (Nuprep, Weaver and Company, Aurora, CO) (6). Electrode impedances were maintained below $10 \mathrm{kOhms}$ as verified by the EL-Check electrode checker (Biopac Systems Inc., Goleta, CA).

For the MVIC procedure, positioning was identical to the standard bench press but with the pressing apparatus configured to restrict the participant to a 90-degree elbow angle. Participants performed 3 sustained pulses of maximal isometric contractions for approximately 3 seconds separated by another 3 -second pause. The highest recorded value in each muscle for any of the three contractions was used as the normalization value for subsequent EMG data (6).

Following the MVIC test, participants performed 3 bench press repetitions at $75 \%$ of their 1RM in each of the three aforementioned testing conditions: standard (figure 1a), leg-drive (figure 1b), and feet-in-the-air (figure 1c). The 3 repetitions for each randomly ordered condition were performed to a metronome set at 60 beats per minute to standardize lifting velocity across participants. Starting with the barbell in the upward position the participant began the eccentric phase of the lift on a metronome beep, reached the bottom of the lift at the next beep, started the concentric movement on the third beep, and finished the rep on the fourth beep. This procedure was repeated for the following 2 reps. Participants had 4 minutes of rest between conditions. If any of the trials failed to meet the metronome timing, participants were given 4 minutes of rest and the trial was repeated. During the standard and leg-drive conditions, the right leg of the participant was positioned on a force plate to record vertical and antero-posterior GRF data.

The raw EMG data were collected and processed through Biopac's Acknowledge 4.4 software (Biopac Systems Inc., Goleta, CA). Data were band pass filtered at 10-500 Hz, full wave rectified, and smoothed using a root mean square (RMS) moving window of $100 \mathrm{~ms}$ (6). The processed EMG data were then exported to Microsoft Excel for calculation and extraction of the variables of interest. The average of the peak EMG muscle activations, across the 3 reps for each condition, was calculated and then normalized to the peak muscle activation value from the MVIC. Thus, normalized muscle activation values are presented as a percentage of MVIC.

The GRF data were collected in AMTI's NetForce software (AMTI Inc., Watertown, MA) and exported to be used in Matlab (v. R2017b, Mathworks, Natick, MA) for processing. Ground reaction force data were smoothed using a $4^{\text {th }}$ order Butterworth filter with a cutoff frequency set at $20 \mathrm{~Hz}$ (16). Peak vertical and antero-posterior GRF were determined for each rep and then averaged across reps for the leg-drive condition. The standard bench press condition did not result in 3 distinct peaks like in the leg-drive condition, thus, for the standard condition, the maximum GRF values were used for analysis. The GRF values were then normalized to body weight to determine the percentage of body weight at which the participant was pressing.

\subsection{Statistical Analyses}

For the EMG data, between group and within group differences for peak muscle activations were analyzed using a $2 \times 3$ (gender $\times$ condition) analysis of variance (ANOVA). In the presence of a significant F-value, a Bonferroni post-hoc analysis was conducted to determine where, if any, differences occurred. The criterion level for significance was set at $p \leq 0.05$.

In addition, averaged peak vertical and antero-posterior GRF variables were analyzed using a 2 × 2 (gender $\mathrm{x}$ condition) ANOVA. This only included 2 conditions because force plate data was not collected for the feet-in-the-air condition. The criterion level for significance was set at $p \leq 0.05$. In the presence of a significant finding, 95\% confidence intervals $(95 \% \mathrm{CI})$ and Cohen's D effect sizes (ES) were reported. Cohen's D effect sizes were interpreted as $0.20=$ small, $0.50=$ moderate, and $0.80=$ large (2).

\section{Results}

Figure 2 shows mean muscle activation values, in \% MVIC, for each of the three muscles, across gender and condition. The results of the ANOVA revealed no statistically significant differences across gender or condition for any of the three muscles analyzed (pectoralis major gender $p=0.939$, condition $p=0.405$; anterior deltoid gender $p=0.734$, condition $p=0.297$; triceps brachii gender $p=0.456$, condition $p=0.092$ ). Graphically, there is a trend with the feet-in-the-air condition resulting in higher muscle activations than the other two conditions. This is only a noted trend and not substantiated statistically by the ANOVA. 


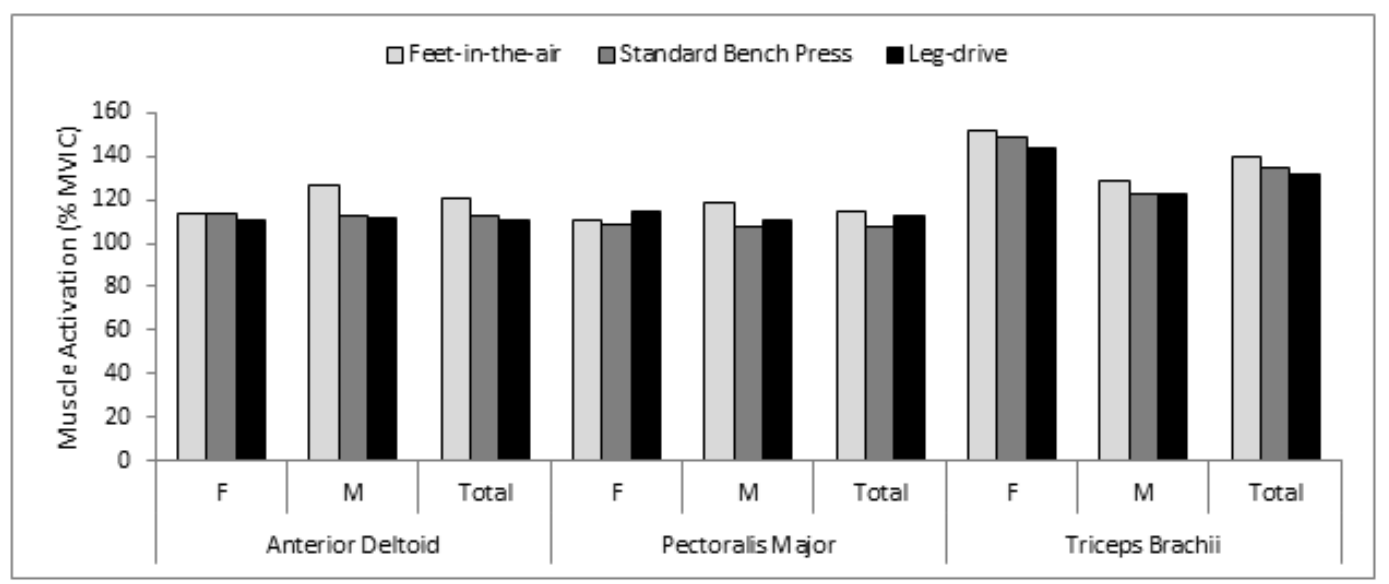

Figure 2. Mean muscle activation values normalized to MVIC across gender and condition. There were no statistically significant differences found across any of the conditions.

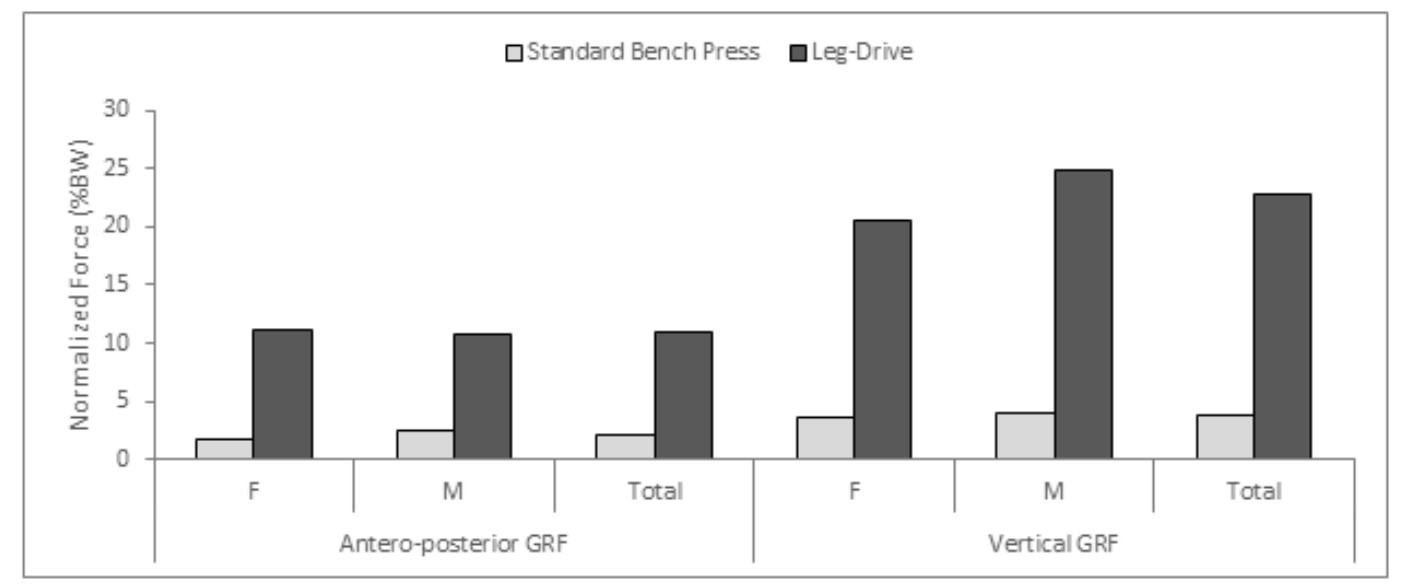

Figure 3. Peak (for standard condition) and mean peak (for the leg-drive condition across the three reps) antero-posterior and vertical ground reaction forces normalized to body weight. Note, no data are presented for the feet-in-the-air condition because the feet were not in contact with the ground. All condition comparisons were found to be statistically significant at the $\alpha=0.05$ level.

Figure 3 shows the mean GRF data across gender and conditions. The results of the ANOVA for the GRF data revealed a significant increase in both vertical (mean difference $=19.03 \% \mathrm{BW}, 95 \% \mathrm{CI}=16.46-21.30 \% \mathrm{BW}, p$ $<0.001$, ES $=4.81$ ) and antero-posterior (mean difference $=8.85 \% \mathrm{BW}, 95 \% \mathrm{CI}=7.11-10.60 \% \mathrm{BW}, p<0.001, \mathrm{ES}$ $=6.99) \mathrm{GRF}$ in the leg-drive compared to the standard bench press condition. Additionally, there were no statistically significant differences between genders for the vertical $(p=0.095)$ or antero-posterior $(p=0.528)$ GRF.

\section{Discussion}

The intent of this study was to examine the effects of leg-drive on upper extremity muscle activation during the bench press exercise. It was hypothesized that no differences in muscle activation of the three upper extremity muscles would exist regardless of lower extremity involvement and leg position. The hypothesis was supported in that there were no statistically significant findings in any of the muscle activations of the pectoralis major, anterior deltoid, or triceps brachii during the three conditions of the bench press.

From an observational standpoint, lifters who utilize leg-drive feel as if they are capable of lifting more weight, or, performing a better lift when compared to a standard technique. This is further evidenced by Kristiansen et al. who demonstrated that experienced lifters utilized the vastus lateralis muscle more during a bench press at $75 \%$ of a 3-RM while inexperienced lifters did not (8). The authors suggest that this posture led to a greater strength expression in the experienced lifters. While this current work did not result in a greater strength expression, it is worth noting that Kristiansen et al. did not control the participants' posture on the bench. In their observations, they reported that experienced lifters tended to arch their backs during the lift, while the inexperienced lifters did not. It is probable that the "greater strength expression" they are describing was a result of an increase in the torso angle to 
mimic a decline bench press. For example, Lauver et al. showed that compared to a flat bench press, a decline position results in more utilization of the lower fibers of the pectoralis major and simultaneously places less emphasis on the anterior deltoids (10). A decline position places more demand on the larger musculature of the pectoralis major, which better handles that demand and removes some demand from the smaller musculature of the anterior deltoids.

Within this study, the participants were required to maintain 5 points of body contact (in the leg-drive and standard conditions), one of which was the buttocks on the bench. This variable was controlled in order to minimize the effects of spinal posture in the lift. It is clear from the GRF data that the participants pressed harder into the ground during the leg-drive (anteroposterior $=11.00 \%$ $\mathrm{BW}$, vertical $=22.85 \% \mathrm{BW}$ ) compared to the standard bench press condition (anteroposterior $=2.16 \% \mathrm{BW}$, vertical $=3.82 \% \mathrm{BW}$ ). Thus, any expected changes in muscle activity would be attributed to the leg-drive technique. If leg-drive was easier from a muscle activation perspective, one of two different scenarios may be expected: 1. there might be increased muscle activations with the leg-drive technique, indicating that the positioning would cause the central nervous system (CNS) to recruit more motor units to perform the lift. 2. with leg-drive, the body is placed into a mechanical advantage thereby reducing demand placed on the central nervous system. This would result in less motor unit recruitment necessary for the lift. If the lift were "easier," then this scenario would make more sense, as it would take less muscular effort to lift the given weight. The latter theory is more consistent with Ploutz et al. who showed that after 9 weeks of resistance training, the quadriceps utilized less muscle for a given lift when compared to pre-training (15). Thus, the more a muscle is prepared for, or capable of performing a demanding task, the less effort is required of that muscle to complete that task.

Due to the lack of muscle activation variation, it is plausible that the demand of the task ( $75 \%$ of $1 \mathrm{RM})$ was too light. Since the weight remained at $75 \%$ of $1 \mathrm{RM}$ throughout all conditions, the muscle activation may not have changed because the demands of the weight remained the same. It is possible that increasing the number of reps or the load would tease out muscle activation differences between lifting techniques since the requirement for the CNS would be more demanding. As such, perhaps leg-drive is only beneficial in very heavy, or increasingly demanding loads. Alternatively, it is possible the leg-drive technique yielded no muscle activation difference because the effort to control lifting velocity via metronome hindered the participant's power output.

During the feet-in-the-air condition there was a small trend of higher muscle activation values compared to the other two conditions. While this trend is not statistically significant, it is worthy of mention in light of some studies that show alterations in muscle activity with instability (3, $9,11,12,14,17)$. Current literature on the effects of instability in the bench press is ambiguous. In a study by Nairn et al., participants performed a bench press on a stability ball resulting in increased shoulder muscle activation compared to a standard bench press (12). However, in a study by Saeterbakken, the standard bench press condition resulted in greater EMG activity in the pectoralis major and triceps compared to the unstable conditions (17). The instabilities created in previous studies were either at the level of the bench (e.g. using a stability ball) or at the implement being lifted (e.g. using an earthquake bar). It appears that a majority of the studies resulted in more recruitment of stabilizing muscles rather than the primary movers. Interestingly, in the current study, instability was not purposely created in the bar or the bench in the feet-in-the-air condition, but it appeared that there was some instability in the participants because of not having their feet on the floor to help maintain balance. This small finding may be worth exploring more in future bench press studies.

Since there was not a statistically significant change in EMG activity levels across the conditions, it begs the question, why do lifters feel that performing the leg-drive while lifting is "easier" compared to a standard bench press? In this study, the EMG activity remained at similar levels between all three conditions, but it is important to note that the resistance also remained constant at $75 \%$ of the participant's 1RM. It is possible that since the weight being lifted is the same amount across all conditions, the CNS only recruited the motor units necessary to perform that task; no more, no less. A study by Korak et al. corroborates this idea, noting that the strength and neural activations between two groups performing bench press using the same load (one utilizing rest-pause method and one performing a traditional bench press) were not significantly different (7). Interestingly, they did find that over a 4-week training period, even though EMG activities were not different between groups, the group that was trained in the rest-pause method did increase their lifting volume compared to the traditionally trained group. Since there was not a difference in muscle activity across conditions, it is clear, at least from a muscle activation standpoint that the use of leg-drive while keeping the buttocks on the bench, does not lead to a reduction in motor unit recruitment. However, it is still unknown if leg-drive could feasibly contribute to an increased maximum load lifted or training volume. More research is necessary to determine the effectiveness of this technique.

Future research should examine the effects of leg-drive utilizing a higher or lower percentage of the participant's 1RM.Currently, no studies have examined the effects of leg-drive on any type of performance increases, such as increasing the load or the number or reps during the bench press. Examining this in a future study may reveal if a heavier or lighter weight with leg-drive results in a change 
in muscle activation compared to a standard bench press. Future training studies could compare an intervention group utilizing leg-drive to a control group performing a standard bench press to determine if the maximum amount of weight lifted or volume lifted, would be different over several weeks of training.

\section{Practical Applications}

Based on the findings of the present study, recreational athletes or individuals utilizing the bench press in their training could use the leg-drive technique if they prefer it, or refrain if they do not. Because there were no significant differences in upper extremity EMG activations found between conditions, strength and conditioning coaches or practitioners should base the decision to utilize the leg-drive on the athlete's personal preference.

\section{Acknowledgements}

No external financial support was received for this research. The authors would like to thank all of the volunteers who participated in the study.

\section{REFERENCES}

[1] Barnett, C., Kippers, V., \& Turner, P. Effects of Variations of the Bench Press Exercise on the EMG Activity of Five Shoulder Muscles. Journal of Strength and Conditioning Research. 9: 222-227. 1995.

[2] Cohen, J. Statistical power analysis for the behavioral sciences. Rev. ed. New York: Academic Press, 1977.

[3] Dunnick, D.D., Brown, L.E., Coburn, J.W., Lynn, S.K., \& Barillas, S.R. Bench Press Upper-Body Muscle Activation Between Stable and Unstable Loads. Journal of Strength and Conditioning Research. 29: 3279-83. 2015.

[4] Haff, G., Triplett, N.T., \& National Strength \& Conditioning Association (U.S.) Essentials of strength training and conditioning. Fourth edition. ed. Champaign, IL: Human Kinetics, 2016.

[5] Hermens, H.J., Freriks, B., Merletti, R., Stegeman, D., Blok, J., Rau, G., Disselhorst-Klug, C., \& Hagg, G. European Recommendations for Surface Electromyography, Results of the SENIAM Project: Roessingh Research and Development.

[6] Konrad, P. The ABC of EMG: A practical Introduction to Kinesiological Electromyography. 1.4 ed. Scottsdale, AZ: Noraxon USA, 2006.

[7] Korak, J.A., Paquette, M.R., Brooks, J., Fuller, D.K., \& Coons, J.M. Effect of rest-pause vs. traditional bench press training on muscle strength, electromyography, and lifting volume in randomized trial protocols. European Journal of Applied Physiology. 2017.
[8] Kristiansen, M., Madeleine, P., Hansen, E.A., \& Samani, A. Inter-subject variability of muscle synergies during bench press in power lifters and untrained individuals. Scandinavian Journal of Medicine and Science in Sports. 25: 89-97. 2015.

[9] Langford, G.A., Mccurdy, K.W., Ernest, J.M., Doscher, M.W., \& Walters, S.D. Specificity of machine, barbell, and water-filled $\log$ bench press resistance training on measures of strength. Journal of Strength and Conditioning Research. 21: 1061-6. 2007.

[10] Lauver, J.D., Cayot, T.E., \& Scheuermann, B.W. Influence of bench angle on upper extremity muscular activation during bench press exercise. European Journal of Sports Science. 16: 309-16. 2016.

[11] Lawrence, M.A., Leib, D.J., Ostrowski, S.J., \& Carlson, L.A. Nonlinear Analysis of an Unstable Bench Press Bar Path and Muscle Activation. Journal of Strength and Conditioning Research. 31: 1206-1211. 2017.

[12] Nairn, B.C., Sutherland, C.A., \& Drake, J.D. Location of Instability during a Bench Press Alters Movement Patterns and Electromyographical Activity. Journal of Strength and Conditioning Research. 29: 3162-70. 2015.

[13] National Strength \& Conditioning Association (U.S.). Certification Commission. Exercise technique manual for resistance training. Third edition. ed. Champaign, IL: Human Kinetics, 2016.

[14] Ostrowski, S.J., Carlson, L.A., \& Lawrence, M.A. Effect of an Unstable Load on Primary and Stabilizing Muscles During the Bench Press. Journal of Strength and Conditioning Research. 31: 430-434. 2017.

[15] Ploutz, L.L., Tesch, P.A., Biro, R.L., \& Dudley, G.A. Effect of resistance training on muscle use during exercise. Journal of Applied Physiology. 76: 1675-81. 1994.

[16] Robertson, D.G.E. Research methods in biomechanics. Champaign, IL: Human Kinetics, 2004.

[17] Saeterbakken, A.H. \& Fimland, M.S. Electromyographic activity and 6RM strength in bench press on stable and unstable surfaces. Journal of Strength and Conditioning Research. 27: 1101-7. 2013. 